Proceedings

\title{
Determination of Organic Micropollutants in Water Using Gas Chromatography-Mass Spectrometry ${ }^{\dagger}$
}

\author{
Marek Król 1,2 and Mariusz Dudziak 2,* \\ 1 Dempol-Eco, ul. Składowa 9, 45-125 Opole, Poland \\ 2 Silesian University of Technology, Faculty of Energy and Environmental Engineering, Institute of Water \\ and Wastewater Engineering, ul. Konarskiego 18, 44-100 Gliwice, Poland \\ * Correspondence: mariusz.dudziak@polsl.pl \\ + Presented at Innovations-Sustainability-Modernity-Openness Conference (ISMO'19), Bialystok, Poland, \\ 22-23 May 2019.
}

Published: 7 August 2019

\begin{abstract}
In this study a determination method has been developed for seven different micropollutants, that were selected to represent different compound groups. The selected compounds were: 4-nonylphenol (4-NP), 4-octylphenol (4-OP), anthracene (Ant), alachlor (Ac), heptachlor (Hc), heptachlor epoxide (Hce), and bis(2-ethylhexyl)phthalate (DEHP). Chromatographic separation and mass spectrometer detection conditions were optimized to achieve the best micropollutants separation and the best detection sensitivity. A calibration curves were created at different calibration levels suited of each type of detection mode (Full Scan and Selected Ion Monitoring) and limits of detection (LOD) and limits of quantification (LOQ) were calculated. Furthermore, recovery values were determined for each compound in spiked water samples at levels equal to $10 \%, 50 \%$, and $90 \%$ of the calibration curve range and compared to other studies in which similar methods of determination were used.
\end{abstract}

Keywords: micropollutants; gas chromatography; mass spectrometry; limits of detection; limits of quantification; determination method

\section{Introduction}

The growing number of different organic micropollutants necessitates the development of universal procedures for the simultaneous determination of those micropollutants in water for research and environmental laboratories. The selected compounds for this study were: 4-nonylphenol (4-NP), 4-octylphenol (4-OP), anthracene (Ant), alachlor (Ac), heptachlor (Hc), heptachlor epoxide (Hce), and bis(2-ethylhexyl)phthalate (DEHP). Most of the concentration levels for the given micropollutants in water do not exceed $1 \mu \mathrm{g} / \mathrm{L}$ [1]. For this reason, the determination methods must be characterized by sensitivity low enough to detect micropollutants at levels below that threshold. To develop such determination methods the important step is to recognize and optimize the parameters that affect determination method sensitivity and viability for laboratory use. Gas chromatography coupled with mass spectrometry detection has been used extensively in determination of many different micropollutants from water samples [2-4]. The sensitivity of the mass spectrometer detector depends to a large extent on the mode in which the spectrometer detector operates. It is accepted that the Full Scan (FS) mode is being used mostly for the micropollutant qualitative identification purposes in the unknown water samples, whereas the Selected Ion Monitoring (SIM) mode is being used in quantitative determination of the identified micropollutants. 
The aim of the research was to study the capabilities of gas chromatography coupled with single quadrupole mass spectrometer using solid-phase extraction method in determination of different micropollutants in water samples.

The first step to develop the determination method was optimizing the GC temperature program, injection volume, injecting method and carrier gas flow rate to obtain the highest possible parameters such as: signal-to-noise ratio (SNR) and resolution between each compound, while keeping the time of the analysis at reasonable levels.

The next step was selecting MS operating parameters such as electron source temperature or gain value as well as Target and Qualifier Ion $m / z$ fragments for SIM analysis for each selected compound, basing on Full Scan single sample analysis and creating calibration curves at six different levels for the SIM detection and five different levels for FS detection.

The last step was preparing water samples by fortifying tap water samples with mixture of selected compounds and recovering them via solid-phase extraction (SPE) on selected non-polar SPE columns for recovery analysis and further determination method sensitivity increase.

\section{Materials and Methods}

\subsection{Compounds}

All compounds were bought as a pure, separate analytical standards from Sigma-Aldrich ${ }^{\circledR}$, from which solution standards were prepared by measuring $10 \mathrm{mg}$ of the given substance with precision of $0.1 \mathrm{mg}$ on the calibrated, certified analytical scale, and dissolved in methanol or acetone.

\subsection{GC-MS Conditions}

Prepared samples were measured on an Agilent 7890B gas chromatograph coupled to a single quadrupole Agilent 5977A MSD mass spectrometer detector. The column used was an Agilent J\&W DB-5MS capillary column (length $20 \mathrm{~m} \times$ diameter $0.250 \mathrm{~mm} \times$ film $0.25 \mu \mathrm{m}$ ). The injector port was set to $250^{\circ} \mathrm{C}$. Injection was done in pulsed splitless mode with 50 psi pressure for 0.3 min and 60 $\mathrm{mL} / \mathrm{min}$ purge flow after $0.31 \mathrm{~min}$. The liner used was Agilent 5190-2293: $900 \mu \mathrm{L}$ (splitless, single taper, and ultra inert).

The optimized temperature program was detailed as follows: the initial temperature was $40^{\circ} \mathrm{C}$ with hold time of one min, then ramped up to $180^{\circ} \mathrm{C}$ at rate of $30^{\circ} \mathrm{C} / \mathrm{min}$, next ramped up to $23^{\circ} \mathrm{C}$ at a rate of $5{ }^{\circ} \mathrm{C} / \mathrm{min}$, then ramped up to $300{ }^{\circ} \mathrm{C}$ at rate of $10{ }^{\circ} \mathrm{C} / \mathrm{min}$. The gas used as a carrier was helium at constant flow of $1 \mathrm{~mL} / \mathrm{min}$.

Full Scan mode operated at range from 50 to $400 \mathrm{~m} / \mathrm{z}$. Selected ion monitoring mode operated as listed in Table 1. Both FS and SIM mode ion source operated at $230{ }^{\circ} \mathrm{C}$, with a transfer line temperature of $250{ }^{\circ} \mathrm{C}$, a quadrupole temperature of $150{ }^{\circ} \mathrm{C}$, and a fixed electron energy of $70 \mathrm{eV}$. Before the analysis, GC-MS was tuned with an autotune procedure, using a perfluorotributylamine (PFTBA) standard.

Table 1. Selected compounds fragments used in Selected Ion Monitoring detection operating mode.

\begin{tabular}{ccccc}
\hline Compound & $\begin{array}{c}\text { Target Ion }^{1} \\
(\mathbf{m} / \mathbf{z})\end{array}$ & $\begin{array}{c}\text { Qualifier Ion }^{2} \\
(\mathbf{m} / \mathbf{z})\end{array}$ & $\begin{array}{c}\text { Qualifier Ion }^{3} \\
(\mathbf{m} / \mathbf{z})\end{array}$ & Gain \\
\hline 4-NP & 107 & 220 & 77 & 10 \\
4-OP & 107 & 206 & 77 & 10 \\
Ant & 178 & 176 & 89 & 10 \\
Ac & 188 & 160 & 146 & 10 \\
Hc & 272 & 100 & 237 & 10 \\
Hce & 353 & 81 & 237 & 10 \\
DEHP & 149 & 167 & 104 & 10 \\
1,2,3,4-TCN & 266 & 264 & 194 & 10 \\
\hline
\end{tabular}

${ }^{1}$ Dwell time: $70 \mathrm{~ms},{ }^{2}$ Dwell time: $50 \mathrm{~ms},{ }^{3}$ Dwell time: $30 \mathrm{~ms}$. 


\subsection{Sample Preparation}

\subsubsection{Water Sample Fortification}

Water samples were prepared by adding small amount of the mixed micropollutants standard solution to $100 \mathrm{~mL}$ of tap water, with addition of $1 \%$ methanol in a volumetric flask. The flask was then fairly mixed and shortly after, the prepared sample went under the extraction.

\subsubsection{Sample Extraction}

Prepared water samples were extracted using Supelco Supelclean ${ }^{\mathrm{TM}}$ LC-18 $6 \mathrm{~mL}$ columns containing $500 \mathrm{mg}$ of non-polar, octadecyl bonded, endcapped silica.

SPE cartridges were conditioned with $5 \mathrm{~mL}$ of methanol, followed by $5 \mathrm{~mL}$ of dichloromethane/ethyl acetate $(1: 1, v / v)$, then again $5 \mathrm{~mL}$ of methanol and $5 \mathrm{~mL}$ of demineralized water. Samples were then passed through the SPE cartridges at a flow rate of $5 \mathrm{~mL} / \mathrm{min}$. Next the cartridges were rinsed with $5 \mathrm{~mL}$ of demineralized water and dried by passing air using vacuum for $20 \mathrm{~min}$. The analytes were eluted using $5 \mathrm{~mL}$ of dichloromethane/ethyl acetate $(1: 1, v / v)$ followed by $5 \mathrm{~mL}$ of acetone. The eluted extract was evaporated at $30{ }^{\circ} \mathrm{C}$ under nitrogen flow using a Biotage ${ }^{\circledR}$ TurboVap ${ }^{\circledR}$ Classic II to a volume under $250 \mu \mathrm{L}$, avoiding dryness then $2 \mathrm{~mL}$ of hexane was added and evaporated to a volume of $500 \mu \mathrm{L}$. Samples were then transferred to a $1 \mathrm{~mL}$ volumetric vial, spiked with 1,2,3,4-tetrachloronaphthalene (1,2,3,4-TCN) internal injection standard at levels of 100 ng for SIM analysis samples and $200 \mathrm{ng}$ for FS analysis samples, then filled with hexane.

\section{Results}

First step to develop the determination method was optimizing the GC parameters. The GC temperature gradient affects parameters such as: signal retention time (RT), signal-to-noise ratio (SNR), full width at half maximum (FWHM), thus the resolution (RS). For this case, the simple temperature program was chosen: starting temperature of $80^{\circ} \mathrm{C}$ with six min hold time, steady ramp up temperature to $260{ }^{\circ} \mathrm{C}$, at the rate of $5{ }^{\circ} \mathrm{C} / \mathrm{min}$, then ramp up to $300{ }^{\circ} \mathrm{C}$ at the rate of $20^{\circ} \mathrm{C} / \mathrm{min}$ with three min hold time. Injection of a $1 \mu \mathrm{L}$ of the test sample in methanol at a concentration of a 10 $\mathrm{mg} / \mathrm{L}$, with MS operating in the FS mode resulted in a chromatogram with fully separated compounds of which last was DEHP with retention time of $42.746 \mathrm{~min}$. The results were not satisfactory as the retention time of the DEHP could have been a lot shorter. The prepared sample in methanol was analyzed multiple times at many different temperature program configurations to achieve the shortest analysis time, while maintaining the highest possible SNR, and RS parameters. Finally, the injection volume and pressure was set to $2 \mu \mathrm{L}$ and 50 psi to achieve better detection signal responses and smaller FWHM values. The solvent change to a less polar solvent appeared to be mandatory, since the methanol at such volume and pressure could not form a stable layer of adsorbed compounds on DB-5-MS capillary column, resulting in distorted signals. Changing the solvent to a hexane solved the problem and increased the sensitivity of the analysis at a lower micropollutants concentrations. From the FS analysis the Target and Qualifier fragment ions were selected for the SIM analysis

A calibration curves were prepared for FS and SIM analyses. For FS analysis the calibration curve was created at five concentration levels: 100, 200,300,400, $500 \mu \mathrm{g} / \mathrm{L}$, that correspond to working concentration levels of 1, 2, 3, 4 and $5 \mu \mathrm{g} / \mathrm{L}$. For SIM analysis the calibration curve was created at six concentration levels: 1, 5, 10, 50, 100 and $200 \mu \mathrm{g} / \mathrm{L}$, that correspond to working concentration levels equal to $0.01,0.05,0.1,0.5,1$ and $2 \mu \mathrm{g} / \mathrm{L}$.

For both FS and SIM acquisition methods, limits of detection (LOD) and limits of quantification (LOQ) were calculated as method sensitivity parameters. LOD and LOQ values were calculated according to the ISO guide for estimation of LOD and LOQ from calibration samples [5]. The developed determination method was used to analyze the prepared water sample extracts in both SIM and FS modes at three different concentration levels equal to $10 \%, 50 \%$ and $90 \%$ of the calibration curve, using four replicate analyses at each concentration level. The accuracy of the 
determination method was given as a recovery value and listed with LOD and LOQ values in form of a Table 2 .

Table 2. Average recovery values (\%) obtained using the GC-MS method both in full scan (FS) and SIM mode with Relative Standard Deviation values (parenthesis) at three different concentration levels and limits of detection (LOD) and (limits of quantification) LOQ values for both methods.

\begin{tabular}{|c|c|c|c|c|c|c|c|c|c|c|}
\hline \multirow{3}{*}{ Compound } & \multicolumn{5}{|c|}{ FS Mode } & \multicolumn{5}{|c|}{$\begin{array}{r}\text { SIM Mode } \\
\end{array}$} \\
\hline & \multicolumn{3}{|c|}{$\begin{array}{c}\text { Fortification Level }(\mathrm{n}=4) \\
(\mu \mathrm{g} / \mathrm{L})\end{array}$} & \multirow{2}{*}{$\begin{array}{l}\text { LOD } \\
(\mu \mathrm{g} / \mathrm{L})\end{array}$} & \multirow{2}{*}{$\begin{array}{c}\text { LOQ } \\
(\mu g / L)\end{array}$} & \multicolumn{3}{|c|}{$\begin{array}{c}\text { Fortification Level }(\mathrm{n}=4) \\
(\mu \mathrm{g} / \mathrm{L})\end{array}$} & \multirow{2}{*}{$\begin{array}{l}\text { LOD } \\
(\mu g / L)\end{array}$} & \multirow{2}{*}{$\begin{array}{c}\text { LOQ } \\
(\mu g / L)\end{array}$} \\
\hline & 1.8 & 3 & 4.5 & & & 0.2 & 1 & 1.8 & & \\
\hline $4-\mathrm{NP}$ & $87(7)$ & $103(5)$ & $109(2)$ & 0.14 & 0.48 & $81(10)$ & $91(8)$ & $104(13)$ & 0.0054 & 0.0177 \\
\hline $4-\mathrm{OP}$ & $86(8)$ & $96(11)$ & $99(5)$ & 0.15 & 0.49 & $79(4)$ & $85(12)$ & $102(9)$ & 0.0027 & 0.0091 \\
\hline Ant & $95(3)$ & $78(7)$ & $94(12)$ & 0.21 & 0.71 & $71(2)$ & $90(13)$ & $107(5)$ & 0.0032 & 0.0104 \\
\hline Ac & $107(8)$ & $117(5)$ & $122(4)$ & 0.17 & 0.57 & $78(2)$ & 104(16) & $106(13)$ & 0.0028 & 0.0094 \\
\hline $\mathrm{Hc}$ & $70(11)$ & $89(6)$ & $89(4)$ & 0.14 & 0.45 & $45(7)$ & $76(9)$ & $91(7)$ & 0.0024 & 0.0080 \\
\hline Hce & $82(4)$ & $99(4)$ & $95(2)$ & 0.09 & 0.28 & $69(4)$ & $92(16)$ & $95(5)$ & 0.0035 & 0.0115 \\
\hline DEHP & $113(6)$ & $178(9)$ & $145(2)$ & 0.07 & 0.24 & $188(15)$ & $92(5)$ & $108(7)$ & 0.0040 & 0.0133 \\
\hline
\end{tabular}

\section{Conclusions}

GC parameters affect peak surface area, resolution and sensitivity; thus, optimization of these parameters is a crucial step in introducing the determination method to laboratory use. FS detection mode allows the determining of different micropollutants congeners in environmental water samples, but due to a lower signal-to-noise ratio and LOD, LOQ values than the SIM method, it should be only used for qualitative analysis, and further development of the quantitative SIM analysis.

Achieved recovery values suggest that octadecyl $C_{18}$ phase is well-suited stationary phase for SPE extraction of many different organic micropollutants, but the most polar compounds at the lowest concentration level could not be fully recovered. The SPE extraction recovery values varied between $80-120 \%$ in the most cases, with the exception of a highly polar compounds and the lower concentrations and the DEHP, which is problematic due to its ubiquitousness in the environment, even though the extraction parameters were not optimized. This research proves the possibility of simultaneous determination of seven different micropollutants in water samples using the GC-MS method and the SPE extraction. The LOD and LOQ values achieved in the SIM method suggest that the developed determination method gives satisfactory results in terms of sensitivity for research and environmental laboratories purposes as it does not exceed the $0.01 \mu \mathrm{g} / \mathrm{L}$, which is lower than the concentrations that these compounds appear in the different water samples [1].

Author Contributions: M.K. and M.D conceived and designed the experiments; M.K. performed the experiments; M.K. and M.D. analyzed the data; M.D. contributed reagents/materials; M.K. and M.D wrote the paper.

Acknowledgments: The work was carried out as part of the implementation doctorate funded by the Ministry of Science and Higher Education.

Conflicts of Interest: The authors declare no conflict of interest. The founding sponsors had no role in the design of the study; in the collection, analyses, or interpretation of data; in the writing of the manuscript, and in the decision to publish the results.

\section{References}

1. Król, M.; Dudziak, M. Occurrence and determination of selected micropollutants in water environment regulated by directive 2000/60/WE. Inżynieria Ekologiczna 2018, 19, 38-47, doi:10.12912/23920629/86049.

2. Liu, R.; Zhou, J.L.; Wilding, A. Simultaneous determination of endocrine disrupting phenolic compounds and steroids in water by solid-phase extraction-gas chromatography-mass spectrometry. J. Chromatogr. A 2004, 1022, 179-189, doi:10.1016/j.chroma.2003.09.035. 
3. Rianawati, E.; Balasubramanian, R. Optimization and validation of solid phase micro-extraction (SPME) method for analysis of polycyclic aromatic hydrocarbons in rainwater and stormwater. Phys. Chem. Earth 2009, 34, 857-865, doi:10.1016/j.pce.2009.07.003.

4. Okumura, T.; Nishikawa, Y.; Yamamoto, H.; Konishi, H. Gas chromatography-mass spectrometric determination of fthalide and heptachlor epoxide in environmental samples. Talanta 1997, 44, 649-656, doi:10.1016/S0039-9140(96)02097-8.

5. International Organization for Standardization. Capability of Detection-Part 2: Methodology in the Linear Calibration Case (ISO 11843-2:2000). 2016. Available online: https:/www.iso.org/standard/20186.html (accessed on 3 April 2019).

(C) 2019 by the authors. Licensee MDPI, Basel, Switzerland. This article is an open access article distributed under the terms and conditions of the Creative Commons Attribution (CC BY) license (http://creativecommons.org/licenses/by/4.0/). 